Z Rheumatol $2011 \cdot 70: 7-8$

DOI 10.1007/s00393-010-0710-5

(c) Springer-Verlag 2011

\author{
B. Swoboda ${ }^{1} \cdot$ P. Villiger ${ }^{2} \cdot$ J. Braun ${ }^{3}$ \\ ${ }^{1}$ Orthopädisch-Rheumatologische Abteilung der Friedrich-Alexander-Universität \\ Erlangen im Waldkrankenhaus St. Marien gGmbH, Erlangen \\ ${ }^{2}$ Klinik für Rheumatologie und Klinische Immunologie/Allergologie, Inselspital, Bern \\ ${ }^{3}$ Rheumazentrum Ruhrgebiet, Herne
}

\title{
Elektive Rheumachirurgie aus Sicht des Rheumaorthopäden und des internistischen Rheumatologen
}

blematik dar, die gut informierte Patienten häufig von sich aus ansprechen. Aus diesem Grund widmet sich ein Beitrag der Frage, ob und wie lange vor einer geplanten Operation eine Therapie mit Biologika pausiert werden muss.

\section{Fachkompetenz und Interdiszipli- narität als Voraussetzung einer optimierten Patientenversorgung}

Während sich im Jahre 2007 ein Schwerpunktheft mit dringlichen, nicht aufschiebbaren Operationsindikationen befasste, widmet sich dieses Heft der elektiven Rheumachirurgie. Diese kann grundsätzlich in gelenkerhaltende und gelenkersetzende Eingriffe eingeteilt werden. Nach wie vor droht den Patienten bei persistierender Entzündungsaktivität die nichtreversible Zerstörung von Gelenkstrukturen. Da der künstliche Gelenkersatz „nur“ als Ultima Ratio verstanden werden sollte, darf der richtige Zeitpunkt für gelenkund funktionserhaltende rheumachirurgische Eingriffe nicht versäumt werden. Voraussetzung hierfür ist, dass der internistische Rheumatologe über fundierte Kenntnisse zu den verschiedenen Operationsverfahren verfügt und ihre Bedeutung für den Patienten einschätzen kann. Die endgültige Indikationsstellung liegt natürlich in der Hand des operativ tätigen orthopädischen Rheumatologen. Dieser sollte nicht zu sehr auf bestimmte Operationen spezialisiert sein, da die Fokussierung auf einzelne Körperregionen wie z. B. isoliert die Schulter-, Ellenbogen-, Hand- oder Fußchirurgie die Gefahr birgt, dass die Versorgung des Patienten entsprechend der Spezialisierung erfolgt und sich nicht an den vorrangigen Erfordernissen bzw. Bedürfnissen des Rheumakranken orientiert.

Eine entsprechende orthopädischrheumatologische Fachkompetenz ist über die in Deutschland fast flächendeckend etablierten regionalen Rheumazentren sowie in vergleichbar interdisziplinär agierenden Einrichtungen anderer Länder allgemein zugänglich. Die in solchen Zentren aktiv mitarbeitenden Rheumaorthopäden verfügen über ausreichende Kenntnisse hinsichtlich der neuen medikamentösen Therapien, damit operative Interventionen sinnvoll in mittel- und langfristige Therapiekonzepte eingebunden werden können. Um der interdisziplinären Herausforderung hinsichtlich der Indikationsstellung und auch des perioperativen Managements gerecht $\mathrm{zu}$ werden, wurden die Beiträge von internistisch- und orthopädisch-rheumatologisch tätigen Kollegen verfasst, die im klinischen Alltag regelmäßig mit diesen Fragen zu tun haben.

Nicht berücksichtigt werden in diesem Themenheft der künstliche Gelenkersatz an Hüfte und Knie, obwohl dies natürlich die inzwischen am häufigsten durchgeführten orthopädischen Interventionen bei Patienten mit entzündlichen und nichtentzündlichen rheumatischen Erkrankungen sind. Ein Grund hierfür ist, dass sich aktuelle Diskussionen weniger auf die Indikationsstellung als auf operationstechnische Details wie minimale Invasivität beim Zugang und minimale 
Knochenresektion konzentrieren. Leider sind diese in der Öffentlichkeit geführten Diskussionen nicht immer wissenschaftlich fundiert und adressieren nicht die realen Probleme und Risiken. Auch wenn altersbereinigte Statistiken mit einer jährlich steigenden Zahl implantierter Hüftund vor allem Knieendoprothesen bei den Kostenträgern kritische Fragen zur Indikationsstellung provozieren, ist davon auszugehen, dass die Operationsindikation bei den klassischen sekundären Arthritis-Arthrose-Formen an Hüfte und Knie bei Rheumakranken nicht angezweifelt wird. Unter diesen Gesichtspunkten haben wir uns in diesem Themenheft für andere und die aktuelle Rheumaorthopädie relevantere Inhalte entschieden.

\section{Der Fuß - ein vernachlässigter Skelettabschnitt}

Ein nach wie vor vernachlässigter Skelettabschnitt ist der im Schuh gut versteckte Fuß, obwohl nach langjährigem Verlauf bei fast allen Patienten mit rheumatoider Arthritis Pathologien zu finden sind. Nicht nur Clavi bzw. Druck- und offene Stellen mit der Gefahr des Keimeintritts in die Blutstrombahn erfordern eine adäquate orthopädieschuhtechnische Versorgung oder operative Sanierung, sondern auch Synovektomien im Rückfußbereich wie z. B. der Tibialis-posterior-Sehne können einem Kollaps des Längsgewölbes und einem rheumatischen KnickSenk-Fuß vorbeugen. Parallel hierzu erfolgt eine Darstellung operativer Behandlungsmöglichkeiten an der Hand.

\section{Synovektomie und/oder Radiosynoviorthese?}

Obwohl innovative Biologika-Therapien die Notwendigkeit früher Synovektomien großer Gelenke vermindern, können diese Eingriffe bei Patienten mit lokal persistierender Entzündungsaktivität trotz maximaler antiinflammatorischer Medikation immer noch notwendig und indiziert sein. Als konkurrierendes Verfahren wird neben der Chemosynoviorthese häufig die Radiosynoviorthese dargestellt. Hierbei handelt es sich um ein grundlegend differentes nuklearmedizinisches Therapieverfahren. Auch wenn die Indikation grundsätzlich unter Beteiligung des internistischen und/oder des orthopädischen Rheumatologen erfolgen sollte, werden die Grundlagen hier aus Sicht des Nuklearmediziners präsentiert.

Wir hoffen, dass der hier präsentierte Themenschwerpunkt unter dem Gesichtspunkt der Interdisziplinarität Anregungen bietet, dass Rheumakranke aus der Vielzahl an Behandlungsmöglichkeiten zum richtigen Zeitpunkt die richtige Therapie erhalten und somit noch besser versorgt werden können.

Ihre

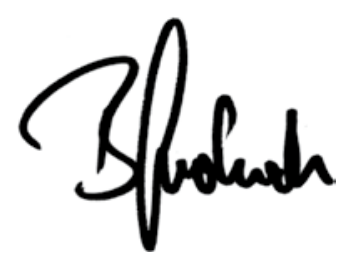

Bernd Swoboda

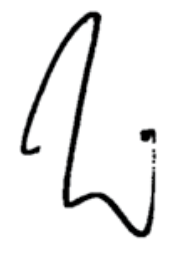

Peter Villiger

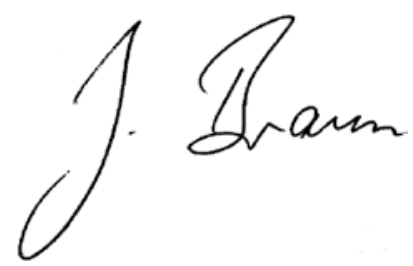

Jürgen Braun

\section{Korrespondenzadresse}

Prof. Dr. B. Swoboda

Orthopädisch-Rheumatologische Abteilung der Friedrich-Alexander-Universität Erlangen im Waldkrankenhaus St. Marien gGmbH Rathsberger Str. 57, 91054 Erlangen sekretariat@ortho-rheuma.med.uni-erlangen.de

\section{Ausschreibung der Forschungsförderung 2011 durch die Sarkoidose Stiftung}

Die gemeinnützige Sarkoidose Stiftung schreibt für 2011 die finanzielle Förderung von Forschungsvorhaben zur Sarkoidose und/oder andere granulomatöser Erkrankungen aus.

Es sollen Forschungsvorhaben gefördert werden, die sich der Grundlagenforschung oder der klinisch orientierten Erforschung dieser Krankheitsbilder widmen. Es können Personal-, Sach- und Reisekosten bis zu einer Gesamtsumme von 10.000,- Euro gefördert werden, die in der Regel innerhalb eines Jahres verausgabt werden sollten.

\section{Der Antrag besteht aus:}

- einer auch für Fachfremde verständlichen Projektskizze auf nicht mehr als 10 Seiten,

- einer einseitigen Zusammenfassung zum Thema,

- dem Lebenslauf,

- dem Verzeichnis bisheriger Veröffentlichungen,

- ggf. bereits abgewickelte Forschungsprojekte.

Dem Antrag können zusätzliche Unter lagen zur näheren Darlegung des Forschungsvorhabens beigefügt werden.

Die Bewerber werden gebeten, ihren Antrag bis zum 31.03.2011 (Poststempel) in zweifacher Ausführung per Post oder als Wordbzw. PDF-Datei per E-Mail bei der Sarkoidose Stiftung einzureichen:

Sarkoidose Stiftung

Geschäftstelle

Uerdinger Str. 43

D-40668 Meerbusch

E-Mail: forschung@sarkoidose-stiftung.de 